\title{
ESTIMACIÓN DE LA EDAD DE LOS CABALLOS BASADO EN EL EXAMEN DENTARIO
}

\section{ESTIMATION OF HORSE AGE BASED ON DENTAL EXAM}

\begin{abstract}
José A. Cardona Á. ${ }^{1}$, Jaime Álvarez P. ${ }^{2}$
${ }^{1}$ MVZ, Esp, M.Sc. Profesor Asociado. Área de Clínica Medico-Quirúrgica de Grandes Animales. Facultad de Medicina Veterinaria y Zootecnia. Universidad de Córdoba. Montería (Córdoba). cardonalvarez@hotmail.com. ${ }^{2}$ MVZ, Esp, M.Sc. Profesor Asociado. Área de Morfofisiología. Facultad de Medicina Veterinaria y Zootecnia. Universidad de Córdoba. Montería (Córdoba).
\end{abstract}

Rev. U.D.C.A Act. E Div. Cient. 13 (1): 29-39, 2010

\section{RESUMEN}

La estimación de la edad de los caballos basado en el examen de los dientes es una labor poco aplicada y limitada por los Médicos Veterinarios, quizás por desconocimiento de los parámetros establecidos en la cronología dentaria equina. Sin embargo, es la forma más adecuada de conocer o verificar la edad de los caballos, aunque tengan o no registros establecidos. En el presente trabajo, se hace una revisión de aspectos relevantes en la dentadura de los equinos, con el fin de conocer y determinar la edad de los ejemplares examinados. Se describe, en forma detallada la estructura del diente, los tipos de dientes, la formula dentaria de los caballos y su evolución en el tiempo, con base en los eventos encontrados.

Palabras clave: Cronología, dientes, equinos

\section{SUMMARY}

Estimation of horse age based on the teeth exam is a work seldom applied and limited by the Medical Veterinaries, due perhaps to ignorance of the established parameters in the equine dental chronology. However, it is the best-suited form to know or to verify the horse's age, although they have or not established records. In the present work a revision of relevant aspects in the denture of the specimens in order to know and to establish the horses age is made. A detailed description of the tooth structure is realized, as well as of the dental formula and its evolution in time.

Key words: Chronology, dents, equine.

\section{INTRODUCCIÓN}

Conocer la edad de los caballos tiene importancia desde el punto de vista clínico y zootécnico. En términos económicos, el valor de un caballo puede estar influenciado por su edad. A través de la observación externa del equino, examinamos sus características generales, su tamaño, su contextura, su temperamento y la presencia de canas, aspectos importantes que se deben tener en cuenta, convirtiéndose, sin embargo, en un método inexacto y subjetivo, debido a que muchos caballos con problemas de salud muestran una edad diferente a la real. Por otra parte, la edad del ejemplar orienta hacia cuáles pueden ser los cuidados que necesita, dependiendo de la etapa en que se encuentre. Los dientes son órganos de consistencia dura, por lo que son bastante resistentes al paso del tiempo, adquiriendo mucha importancia para determinar la edad, con la evaluación de los incisivos presentes, su erupción, su desgaste, su rasamiento y la forma que toma la superficie oclusal o tabla dentaria. En la actualidad, existen métodos sofisticados de identificación y de registro de los animales, por lo que se podría pensar que la estimación de la edad por la dentadura es obsoleta y de poca aplicabilidad; sin embargo, en ausencia de 
registros, dudas o fraudes, este método es la única forma establecida y confiable de determinar la edad en los caballos. Hace muchos años, para determinar la edad, se basaban en métodos empíricos, establecidos por campesinos y vaqueros, quienes utilizaron como parámetro, el número de pliegues que se formaban en el labio y nariz, como el número de años que tenía el animal. De igual forma, los gitanos sabían que, la presencia del colmillo servía para determinar la diferencia entre un caballo joven y uno adulto, así como la desaparición del infundíbulo en la medida que avanzaba la edad, aun que en forma imprecisa e inexacta.

Por experiencias adquirida en el servicio Clínico Ambulatorio de Grandes Animales de la Facultad de Medicina Veterinaria y Zootecnia, de la Universidad de Córdoba, se constató la importancia de determinar la edad de los equinos, basado en su examen dentario. Esto a raíz de que en la mayoría de las explotaciones agropecuarias, no se cuenta con datos o registros que indiquen la edad de sus animales; solo se dispone de la apreciación de los propietarios o de sus manejadores, por lo que para efectos de determinar la edad e incluirla en la historia clínica, se utilizó, como herramienta el análisis de la dentadura aquí descrita y detallada.

Proveer información que sirva como guía a estudiantes y docentes para analizar la dentadura de los caballos, de esta manera poder determinar su edad en forma exacta y precisa en animales durante las prácticas de campo, en exposiciones caninas y en el ejercicio profesional, fueron los objetivos básicos de la presente revisión.

\section{METODOLOGÍA}

Se recopilaron y analizaron datos de literatura científica publicados en revistas indexadas de Estados Unidos, México, España, Brasil, y Argentina, unidos al análisis de datos obtenidos a través de la experiencia en el ejercicio profesional y académico en animales del trópico bajo, el cual posee condiciones ambientales y de manejo diferentes a aquellas donde se desarrollaron los estudios consultados.

\section{ESTRUICTURAS DEL DIENTE}

Los dientes son estructuras papilares duras, intensamente calcificadas, sujetas al maxilar superior e inferior; funcionalmente, son órganos de presión y masticación, así como instrumento de defensa (Scoggins, 2001; Rucker, 2003). En su conformación, cada diente consta de tres partes la corona, el cuello y la raíz. La corona, es la porción que sobresale de la encía (corona clínica) o aquella parte cubierta por el esmalte (corona dental) y puede tener forma triangular o cilíndrica. El cuello, es el segmento de transición entre la corona y la raíz, siendo esta última, la parte del diente alojada en el alveolo mandibular, donde su extremidad inferior presenta una abertura denominada foramen apical o ápex, por donde pasan los vasos y nervios (Kilic et al. 1997a; Tremaine, 1997; Bennett, 2001; Dixon, 2002a; Mitchell et al. 2003).

El diente está constituido de sustancias duras, el esmalte, el cemento, el marfil o dentina y de sustancias blandas, la pulpa dentaria y el periodonto. El esmalte constituye una capa de grosor variable, que cubre la dentina de la corona de los dientes; se distingue por su limpieza, su densidad y su blancura y posee un alto porcentaje de minerales tipo fosfatos y fluoruro de calcio. El cemento es una sustancia similar a la del hueso compacto, la más externa que recubre la raíz del diente, constituyéndose en una capa incrustada sobre la dentina de las raíces, en todos los dientes. El marfil o dentina es un tejido óseo duro, blanco amarillento producido por los odontoblastos, llamada también sustancia fundamental, ya que es la parte principal del diente; forma parte de la corona y de la raíz, cubriendo la superficie de la pulpa dentaria. Se diferencia, químicamente del hueso, por ser más mineralizada y presentar menor cantidad de sustancia orgánica y carbonato de calcio. La pulpa dentaria es una especie de papila que surge del fondo del alvéolo, para alojarse en el cornete dentario interno, formado por tejido conjuntivo, rica en vasos y nervios. El periodonto es una membrana fibrosa, que sirve de unión entre la cara externa de la raíz y la pared del alveolo (Easley, 1996; Foster, 1996; Kirkland et al. 1996; Kilic et al. 1997b; Lowder \& Mueller, 1998; Jones, 2005).

\section{TIPOS DE DIENTES}

Los equinos son clasificados como heterodontes, según su dentadura, debido a que poseen diferentes tipos o grupos de dientes, tales como incisivos, caninos, premolares y molares; también son catalogados como difiodontos, por poseer dos series de dientes, los temporales y los permanentes. Los dientes temporales, también llamados de leche, caducos o deciduos, 
aparecen en las primeras etapas de la vida y después serán reemplazados. Los dientes permanentes o de hueso, de adultos o definitivos, son los que permanecen en el animal por el resto de su vida (Sandoval, 1976; Agüera \& Sandoval, 1999; Konig \& Liebig, 2005).

Los equinos poseen cuatro tipos de dientes, que de acuerdo a su forma y su posición, se pueden clasificar en incisivos (I), situados delante e implantados en el premaxilar y en la mandíbula y su principal función es la de cortar el pasto. En la tabla dentaria de los incisivos, se observa una cavidad o invaginación del esmalte con más de $1 \mathrm{~cm}$ de profundidad, llamada cavidad dentaria externa, infundíbulo o corneto; los incisivos más centrales se denominan pinzas o palas. Los siguientes, hacia el interior, son los medios o medianos y los más externos, se denominan cuñas, cantos o extremos. Los caninos $(C)$ están situados más atrás y su principal función es la de desgarrar los alimentos; sólo aparecen en la dentición definitiva. El espacio que existe entre los caninos y los premolares, se denomina barra o diastema, siendo particularmente grande cuando los caninos están ausentes. Los premolares (PM) y molares (M), constituyen los lados del arca dental; los premolares se hallan primero y aparecen en ambas series dentales, mientras que los molares surgen solo en la dentición de hueso y sirven para triturar el nutrimento (Getty, 1966; Dyce et al. 1991; Caldeira et al. 2002; Dixon, 2002b).

Para diferenciar los dientes de leche de las hueso, y por consiguiente estimar la edad del ejemplar, se debe tener en mente que los dientes incisivos de leche son más blancos, más largos que anchos, ocupan menor volumen, su superficie oclusal es más ovalada, son lisos, no presentan surcos en la cara labial, tienen un cuello definido y bien marcado en la unión con la encía y tiene el infundíbulo menos profundo. Los dientes incisivos de hueso se contrastan por ser pigmentados de color amarillo a marrón; son más largos que anchos o rectangulares, de mayor volumen y presentan surcos en la cara labial (Richardson et al. 1994; Richardson et al. 1995; Richardson, 1997; Fraústo da Silva et al. 2003).

\section{FÓRMULA DENTARIA}

Cuando se habla de fórmula dentaria, se refiere a la manera de expresar el número de dientes que posee un animal, en forma de quebrados, indicando, en la parte superior, el número de cada tipo de diente en el maxilar y, en el quebrado inferior, la cantidad de dientes en la mandíbula, pero solo de una mitad de la boca; para conocer el número total de dientes es necesario multiplicar los datos por dos. Esta fórmula varía con la edad, es desigual en los dientes de leche y en los permanentes (Frape, 1992; Marvin, 1992; Morales, 1997; Gieche, 2007).

La fórmula dentaria de los equinos depende de la edad y del sexo y, es:

Primera dentición, dientes deciduos, de leche o temporales: 2 (I 3/3, C 0/0, PM 3/3) = 24 dientes, para ambos sexos.

Segunda dentición, permanentes, de hueso o definitivos: machos: 2 (I 3/3, C 1/1, PM 3-4/3-4, M 3/3) = 40-44 dientes; hembras: 2 (I 3/3, C 0/0, PM 3-4/3-4, M 3/3) = 36-44 dientes.

La diferencia entre machos y hembras se debe a que en las yeguas los caninos, generalmente, están ausentes. La variabilidad en el número de premolares es causado, por que en algunos caballos se encuentra, en forma irregular, un vestigio del primer premolar, también llamado "diente de lobo"; este diente puede existir en ambas arcadas, pero es más frecuente en la arcada superior, siendo más pequeño que los demás y con raíces cortas (Scrutchfield et al. 1996; Scrutchfield, 2006; Toit, 2006a; Pimentel, 2007).

\section{BASES GENERALES DE LA CRONOLOGÍA DENTARIA EQUINA}

En la medida que el caballo envejece, la valoración de su dentición y, por ende de su edad, implica numerosos criterios; la estimación, se convierte en un proceso complejo, por lo que en algunos animales, se podrá tasar en forma más precisa que en otros. La determinación de la edad es más confiable en caballos jóvenes, ya que, inicialmente, se observa el tipo de diente presente y la valoración de la etapa de erupción, mientras que los dientes permanentes están en función de la estimación de su desgaste y la determinación de la edad depende, específicamente, de los cambios en la superficie oclusal o tabla dentaria, ya sea por su rasamiento o por la manera que toman con el desgaste, por lo que, a mayor edad, la exactitud de la estimación decrece. Esto, se debe a que las tasas de desgastes son variables y son influenciadas por numerosos factores como, raza, desvíos de 
comportamiento (pica o malacia), actividad, época del año, alteraciones en la conformación ósea (picudos) y tipo de alimento o suplemento, ya que los caballos que pastan en pastos secos o toscos presentan una tasa de desgaste mayor que los que pastan en potreros con pasto tierno y jugoso (Richardson, 1997). Es por esto que, en las condiciones de bosque húmedo tropical, como la costa norte colombiana, existen variaciones ambientales que ocasionan cambios en las condiciones nutricionales y físicas del pasto haciéndolo más duro y seco, obligando a que el animal lo mastique por más tiempo, pudiendo generar mayor desgaste, así como patologías bucales como las aristas dentarias (puntas de muela), hiperplasia del paladar (palatitis o haba) que pueden de alguna manera influir en la evolución dentaria de los caballos.

Para poder estimar la edad de los caballos por su dentadura es preciso conocer, en detalle los conceptos básicos de la cronología dentaria equina y saber manejar apropiadamente a los animales en el proceso de evaluación.

Método para abrir la boca. Para evaluar la boca del equino, se procede a abrirla a través del reflejo palatomaxilar, que consiste en introducir el dedo por detrás del colmillo superior y tocar con suavidad el paladar superior; inmediatamente, el animal dobla la lengua y baja la mandíbula inferior abriendo la boca. Para evaluar mejor los dientes y la cavidad oral es conveniente agarrar y sacar por un lado su lengua (Taylor $\mathcal{E}$ Hillyer, 1999).

Erupción de los dientes incisivos. Nacen en forma secuencial desde las pinzas; luego, los medios y, por último, los extremos. En los incisivos de leche, las pinzas nacen durante la primera semana de vida, los medios entre las 4 y 6 semanas y los extremos ente los 5 y 6 meses de edad y a los 9 meses deben estar todos parejos (Easley, 2008).

Muda de los dientes incisivos. Es el mecanismo de reemplazo de los dientes de leche o temporales por los dientes de hueso o permanentes. Esta etapa inicia a los 2,5 años y termina a los cinco años. Inicia con los incisivos inferiores: primero las pinzas, luego los medios y, por último, los extremos (Toit, 2006b). A los 4,5 años emergen en el macho los colmillos y están completamente desarrollados a los cinco años, coincidiendo esta etapa con la muda de los extremos. Cuando ha mudado todos sus incisivos, se dice que el caballo tiene la boca hecha o de hueso (Caldwell, 2006; Linkous, 2006).

Rasamiento. Es considerado como el inicio del desgaste de la cara oclusal del diente; cuando el desgaste es total, se estima que el diente, a nivel del esmalte central, está nivelado (Real, 1990; Richardson et al. 1994).

Estrella dentaria. También llamada estrella de Girard y se presenta en la medida que el desgaste llega a la cavidad pulpar, apareciendo una nueva capa de dentina. Es de color amarillo pardo y aparece en la superficie oclusal, en el lado de la cara labial y, finalmente, se ubica en el centro; al principio es lineal, luego, se forma ovalada y, por último, circular, reflejando el grado de desgaste de los dientes. Aparece secuencialmente en las pinzas a los cinco años, en los medios, a los seis años, y en los extremos, a los siete a ocho años (Muylle et al. 2002; Klugh, 2006).

Cola de alondra. Conocida como golondrina, gavilán o gancho del extremo superior y se forma en el borde caudal del incisivo superior extremo, en forma de gancho, debido a que el desgaste de esa parte del diente es lento. Cuando los incisivos superiores adquieren su posición oblicua con los inferiores, el extremo en el que se ha formado el gancho vuelve a contactar con el diente opuesto y el gancho desaparece, se habla de la presentación de dos colas de alondras en la vida del caballo, donde la primera aparece a los siete años y la segunda a los once o doce años (Fraústo da Silva et al. 2003).

Surco de Galvayne. Es un surco visiblemente manchado y se observa en la cara labial de los incisivos extremos superiores, en caballos de 10 a 20 años (Richardson, 1997).

Cambios en la forma de las superficies oclusales. Con el aumento del desgaste, que es proporcional a la edad, la tabla dentaria cambia de forma, de elíptica a redonda, luego a triangular y, por último, a oval o biangular (Habel, 1988; Fraústo da Silva et al. 2003).

Vista de perfil de la arcada dentaria. Como consecuencia de la forma y desgaste de los incisivos, la vista de perfil de la oclusión de las arcadas dentarias se altera y se torna 
angulada, haciéndose cada vez más al avanzar la edad del caballo. El ángulo, se torna más agudo a partir de los diez años (Rucker, 1996; 2004; 2006).

En el formato 1, se registran las características dentales, arriba detalladas, lo cual permitirá al Médica Veterinario, estimar de manera concreta la edad del ejemplar que entra a la clínica. Conocer la edad real del paciente, se considera vital para cualquier tratamiento que éste pueda requerir.

\section{SECUENCIA DE LOS EVENTOS EN LA CRONOLOGÍA DENTARIA}

Es importante tener en cuenta que para estimar la edad de los caballos, a través de la evaluación de sus dientes, se debe iniciar el proceso analizando, en forma lógica, la evolución de los eventos, en el tiempo.

Son siete momentos que se dan en la dentadura de los equinos con el correr del tiempo, por lo tanto, en la

Formato 1. Registro dentario en equinos

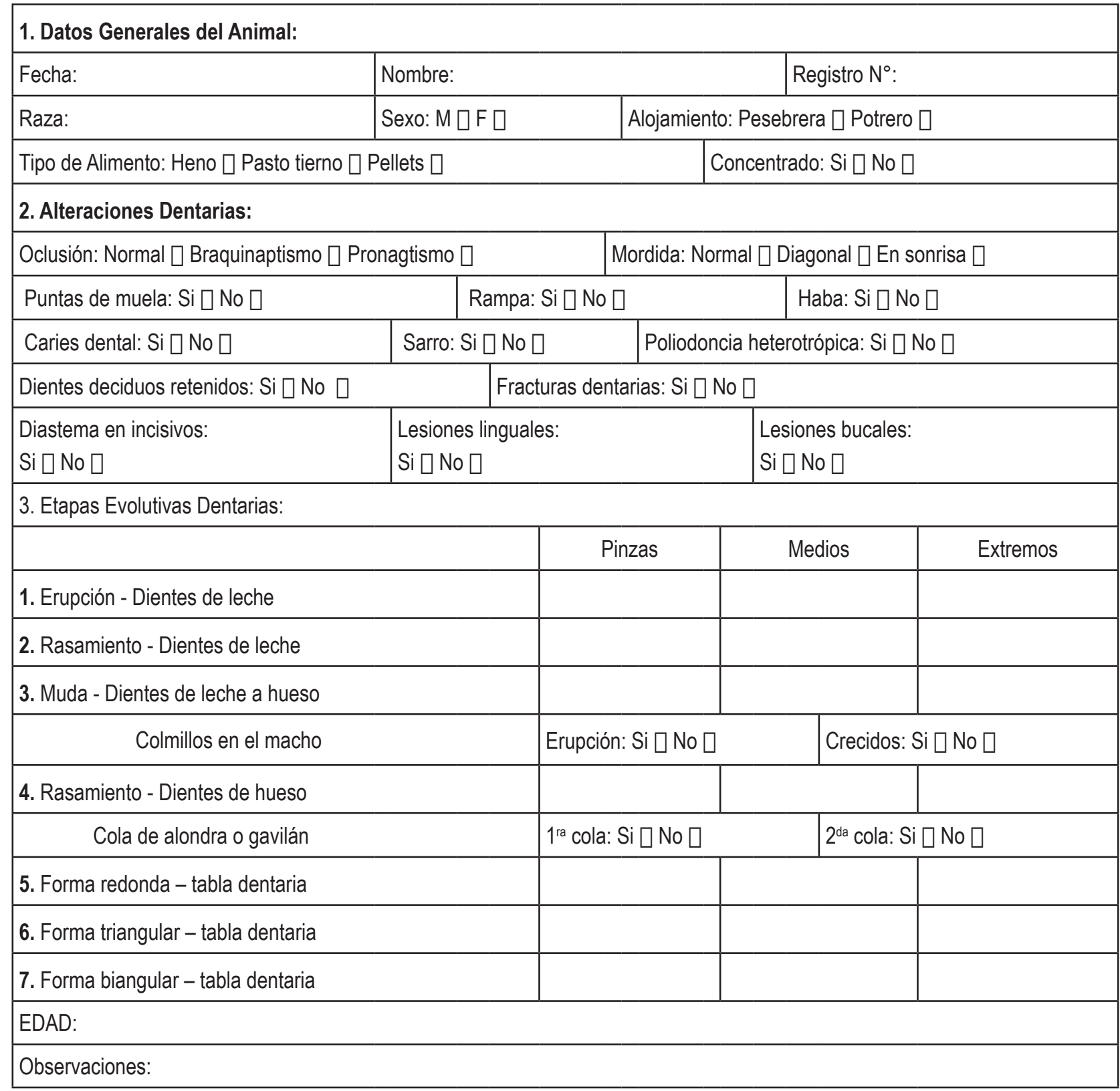


medida que el caballo se hace viejo, va avanzando en las etapas de su dentadura. Todas las fases dentales ocurren en orden secuencial y siempre inicia con los dientes incisivos centrales o pinzas, luego, en los medios y, por último, en los extremos o cuñas. Las etapas que se dan en forma secuencial en la dentadura del caballo son: nacimiento de los dientes temporales (leche), rasamiento de los dientes temporales (leche), muda de los dientes temporales (leche) a permanentes (hueso), rasamiento de los dientes permanentes (hueso), cambio en la forma de la tabla dentaria, que comienza con forma redonda, posteriormente triangular $\mathrm{y}$, al final biangular (Muylle, 2002) (Cuadro 1).

Primera etapa. Consiste en la erupción de los dientes deciduos o de leche, donde emergen las pinzas en la primera semana; luego nacen los medios, entre 30 a 60 días y, finalmente los extremos, a los seis meses. A los nueve meses se encuentran todos los dientes parejos o nivelados con la arcada dentaria (Scrutchfield, 1991; Peña E Herrador, 1991) (Cuadro 2).

Segunda etapa. Es el rasamiento de los dientes de leche, e inician con las pinzas al año, luego, los medios al 1,5 años y los extremos, por último, a los dos años (Fraústo da Silva et al. 2003) (Cuadro 3).

Tercera etapa. Se da la muda de dientes temporales o de leche a dientes permanentes o de hueso. Esta etapa inicia con la muda de los dientes incisivos centrales o pinzas a los 2,5 años y están crecidos o nivelados con la arcada dentaria a los tres años; posteriormente mudan

Cuadro 1. Etapas evolutivas en la cronología dentaria equina.

\begin{tabular}{|c|c|c|c|}
\hline Etapas evolutivas & $P$ & M & $E$ \\
\hline 1. Erupción - Dientes de leche & $1^{\text {ra }}$ semana & 1 - 2 meses & $5-6$ meses \\
\hline 2. Rasamiento - Dientes de leche & 1 año & 1,5 años & 2 años \\
\hline 3. Muda - Dientes de leche a hueso & $2,5-3$ años & 3,5 - 4 años & 4,5 - 5 años \\
\hline Colmillos en el macho & \multicolumn{3}{|c|}{ Nacen a los 4,5 años y crecidos a los 5 años } \\
\hline 4. Rasamiento - Dientes de hueso & 6 años & 7 años & 8 años \\
\hline Cola de alondra & \multicolumn{3}{|c|}{ Se forma a los $7 / 11$ años en extremos superiores } \\
\hline 5. Cambio forma: Forma redonda & 9 años & 10 años & 11 años \\
\hline 6. Forma triangular & 12 años & 14 años & 17 años \\
\hline 7. Forma biangular & 19 años & 20 años & 24 años \\
\hline
\end{tabular}

Adaptado según Peña \& Herrador, 1991; Richardson, 1997; Tremaine, 1997; Martin, 1999; Fraústo da Silva et al. 2003; Linkous, 2006; Toit, 2006a.

Cuadro 2. Erupción y muda de los dientes incisivos en el caballo.

\begin{tabular}{|c|c|c|c|}
\hline \multicolumn{2}{|c|}{ Incisivos de leche } & \multicolumn{2}{c|}{ Incisivos de hueso } \\
\hline & Erupción & Erupción & Nivel de arcada \\
\hline Pinzas & $1^{\text {a }}$ semana & 2,5 años & 3,0 años \\
\hline Medios & $1-2$ meses & 3,5 años & 4,0 años \\
\hline Extremos & $5-6$ meses & 4,5 años & 5,0 años \\
\hline
\end{tabular}

Adaptado según Richardson, 1997; Tremaine, 1997; Linkous, 2006; Toit, 2006 a. 
Cuadro 3. Rasamiento de los incisivos de leche y de hueso en el equino.

\begin{tabular}{|c|c|c|}
\hline \multicolumn{3}{|c|}{ Rasamiento de los incisivos en el equino } \\
\hline & Incisivos de leche & Incisivos de hueso \\
\hline Pinzas & 1,0 año & 6,0 años \\
\hline Medios & 1,5 años & 7,0 años \\
\hline Extremos & 2,0 años & 8,0 años \\
\hline
\end{tabular}

Adaptado según Richardson, 1997; Fraústo da Silva et al. 2003.

los medios a los 3,5 años y se nivelan a los cuatro y, al final, mudan los extremos a los 4,5 años y se nivelan a los cinco. La muda de los extremos coincide con la erupción de los colmillos en el macho, emergen a los 4,5 y están desarrollados a los cinco años. Después de ésta etapa, el caballo posee toda su dentadura de hueso o permanente y se dice que tiene la "Boca hecha" (Baker, 1991; Villanueva-Salcedo, 2001) (Cuadro 2).

Cuarta etapa. Se inicia el rasamiento de los dientes permanentes o de hueso; esta etapa inicia con el rasamiento de las pinzas a los seis años; luego rasan los medios a los siete y, por último, los extremos a los ocho años. A los siete años, se forma la primera cola de alondra o gavilán, que consiste en la formación de un gancho en los extremos superiores (Baker, 1991) (Cuadro 3). La estrella dentaria aparece a los siete años en las pinzas, luego, en los medios a los ocho años y, finalmente, en los extremos, a los nueve años (Martin, 1999; Walmsley, 1993) (Cuadro 4).

Quinta etapa. Consiste en el cambio de forma que toma la cara oclusal o tabla dentaria de los incisivos superiores e inicia cambiando a forma redonda por las pinzas a los nueve años; luego, los medios a los diez años y, por último, los extremos, a los once años. A los diez años aparece una mancha café en el extremo superior, el surco de Galvayne y desaparece a los 20 años. A los once años, se forma la segunda cola de alondra en los extremos superiores (Walmsley, 1993) (Cuadro 5).

Cuadro 4. Aparición de la estrella dentaria y del surco de Galvayne en los incisivos del equino.

\begin{tabular}{|c|c|c|}
\hline & Estrella dentaria & Surco de Galvayne \\
\hline Pinzas & 7,0 años & - \\
\hline Medios & 8,0 años & - \\
\hline Extremos & 9,0 años & $>10$ años $\mathrm{y}<20$ años \\
\hline
\end{tabular}

Adaptado según Richardson, 1997; Fraústo da Silva et al. 2003.

Cuadro 5. Forma que toma la tabla dentaria en los incisivos del caballo.

\begin{tabular}{|c|c|c|c|}
\hline & Forma redonda & Forma triangular & Forma oval \\
\hline Pinzas & 9 años & 13 años & $>19$ \\
\hline Medios & 10 años & 14 años & $>20$ \\
\hline Extremos & 11 años & 17 años & $>24$ \\
\hline
\end{tabular}

Adaptado según Martin, 1999; Fraústo da Silva et al. 2003. 
Sexta etapa. Consiste en la forma triangular que toma la cara oclusal y que inicia con las pinzas a los 13 años; luego, los medios a los 14 años y, finalmente, los extremos a los 17 años (Peña E Herrador, 1991) (Cuadro 5).

Séptima etapa. Se cambia a forma biangular u oval y comienza con las pinzas a los 19 años, los medios a los 20 y los extremos a los 24 años; sin embargo cabe anotar que, a esta edad, muchos equinos han perdido su dentadura (Martin, 1999) (Cuadro 5).

Análisis lógico. Al tratar de establecer la edad del caballo, se debe determinar qué dientes incisivos tiene; luego, si éstos son deciduos, permanentes o de ambos, se analiza las características típicas de cada tipo de hueso; si posee todos sus dientes de hueso, se procede a determinar su rasamiento $\mathrm{y}$, por último, la forma de la tabla dentaria. Este procedimiento debe ser pausado y minucioso para no cometer errores. Por ejemplo, si un equino presenta todos sus incisivos y sólo tiene las pinzas de hueso crecidas y los demás incisivos son de leche, entonces, el caballo tiene 3,0 años, ya que a los 2,5 se mudan las pinzas y están crecidas a los 3,0 años; pero si tuviese las pinzas y medios de hueso ya crecidos y erupcionando los extremos de hueso, indicaría que tiene 4,5 años y coincidiría con la erupción de los colmillos en el macho. Ahora, en un caballo con toda su dentadura de hueso (boca hecha), se determina cuáles de los incisivos están rasando y si se percibe que solo están rasando las pinzas, entonces, tiene seis años. $\mathrm{Si}$, se precisa que ya todos los incisivos de hueso rasan, entonces, se procede a determinar la forma de la tabla dentaria y dependiendo de su forma y qué dientes hacen parte, se concreta la edad; por ejemplo si las pinzas y los medios tienen forma triangular, el caballo tiene 14 años.

Datos obtenidos en el servicio ambulatorio de la Clínica Médico-Quirúrgica de Grandes animales de la Facultad de Medicina Veterinaria y Zootecnia de la Universidad de Córdoba, indican que aproximadamente el $60 \%$ de los equinos examinados (caballos, mulos y burros), no mostraron la edad reportada por sus propietarios, al confrontarla con la edad establecida por su dentadura, existiendo, en la mayoría de los casos (90\%), una diferencia de edad, que oscilaba entre seis meses y un año. El grupo etáreo donde se determinó mayor diferencia de edades a las reportadas fue el que se encontraba entre 4,5 años y 8 años, seguida del grupo, entre 9 y 13 años.
Esta variación, se podría explicar por la falta de registros de los equinos de vaquería, nacidos en la explotación o comprados, sin determinar su edad previamente, por esta razón, se apela a los datos informados por su memoria. Cabe anotar que los caballos utilizados en labores de vaquería están en constante descarte y adquisición, de igual forma, los operarios y encargados de los animales pueden ser cambiados, por lo que se pierde la información al no estar depositadas en registros físicos y establecidos en forma técnica. Por otro lado, los animales que se encuentran en potreros poseen una conducta alimentaria diferente a los que se encuentran en pesebreras, lo que podría influenciar en la variación de la edad reportada con la dentaria.

Los casos en los cuales la edad dentaria correspondía con la edad reportada por sus propietarios (40\%), fueron aquellos que habían nacido en la explotación y tenían registros o aquellos que fueron reportados ante la Federación Nacional Fedequinas, Federación Nacional que expide el registro del caballo. El grupo etáreo donde se determinó la edad, en forma más acertada a las registradas fue, entre 2,5 y 4,5 años.

Se concluye que la cronología dentaria en equinos es un método bastante preciso hasta los ocho años de edad, ya que se fundamenta, principalmente, en la erupción y el rasamiento de los incisivos de leche, la muda de dientes de leche a dientes de hueso y su rasamiento, así como la erupción de los colmillos en el macho. De igual forma, debido al rasamiento de la tabla dentaria, aparecen la cola de alondra y la estrella dentaria; sin embargo, después de los nueve años, se hace inexacta la estimación de la edad, ya que los cambios que se evalúan en la tabla dentaria pueden estar influenciados por varios factores, como el tipo de alimento y la conducta alimentaria, por lo que es preciso tener en cuenta las características específicas de cada individuo, como anamnesis, raza, sexo, alimentación o suplementación, estabulación o pastoreo.

Este método ha sido de mucho valor técnico en el desempeño de las actividades de la clínica de grandes animales de la Facultad de Medicina Veterinaria, para determinar la edad de los caballos. Principalmente, en aquellos ejemplares que se dedican a labores de vaquería en explotaciones ganaderas, ausentes de registros, expuestos a diferentes condiciones de manejo y alimentación, con condiciones corporales deficientes, 
lo que podría hacerlo ver de mayor edad. De allí, es de mucho valor interpretar los cambios que posee en su dentadura y la influencia de factores externos, de esta manera tener datos fehacientes y objetivos sobre la edad real o muy aproximada de los caballos evaluados, evitando de esta manera engaños, fraudes o equivocaciones indeseadas.

\section{BIBLIOGRAFÍA}

1. AGÜERA, E.; SANDOVAL, J. 1999. Anatomía Aplicada del Caballo. Ed. Haurcourt (España). p.38-40.

2. BAKER, G. 1991. Dental Morphology, Function, and Pathology. 37th Am. Assoc. Equine Practitioners. Conference Proc. p.83-93.

3. BENNETT, G. 2001. Bits and bitting: form and function. Proc. Am. Assoc. Equine Practitioners. 47:130-137.

4. CALDEIRA, R.; FRAÚSTO DA SILVA, M.; GRAVE, J.; ROSA, I.G.; MENDONÇA, H. 2002. Apontamentos de Exognosia. Faculdade de Medicina Veterinária, Universidade Técnica de Lisboa. p.18-20.

5. CALDWELL, L. 2006. Canine Teeth in the Equine Patient - The Guide to Eruption, Extraction, Reduction and Other Things You Need to Know. Am. Assoc. Equine Practitioners. Disponible desde Internet en: http://www.ivis.org/proceedings/aaepfocus/2006/ caldwell1.pdf (con acceso 12/07/08).

6. DIXON, P. 2002a. Anatomía Dental. En: Baker, G.; Easley, J. eds. Odontología Equina XXI. Ed. Intermédica (Argentina): p.3-30.

7. DIXON, P. 2002b. The gross, histological, and ultrastructural anatomy of equine teeth and their relationship to disease. 48th Am. Assoc. Equine Practitioners Annual Convention. 48:421-437.

8. DYCE, K.; SACK, W.; WENSING, C. 1991. Anatomía Veterinaria. Ed. Panamericana (Argentina): p.123125.

9. EASLEY, J. 1996. Equine dental development and anatomy. In: In-depth dentristy seminar. Proc. Am. Assoc. Equine Practitioners. 42:1-10.
10. EASLEY, J. 2008. Enfermedades orales y dentales. En: Hinchcliff, K; Kaneps, A; Geor, R; Warrick, B. eds. Medicina y Cirugía en los Equinos de Deporte. Ed. Intermédica (Argentina): p.1205-1215.

11. FOSTER, D. 1996. Nomenclature for equine dental anatomy based on the modified triadan system. $42^{\text {nd }}$ Am. Assoc. Equine Practitioners Annual Convention. p.18-319.

12. FRAPE, D. 1992. Nutrición y Alimentación del Caballo. Ed. Acribia (España): p.3.

13. FRAÚSTO DA SILVA, M.; GOMES, T.; DIAS, A.; AQUINO, J.; MENDES, L.; CAVACO, J.; ALEXANDRE, G.; CALDEIRA, R. 2003. Estimativa da idade dos equinos através do exame dentário. Rev. Port. Cienc. Vet. 98(547):103-110.

14. GETTY, R. 1966. Atlas de Anatomía Práctica Veterinaria Aplicada. Ed. UTEHA (Mexico): p.68

15. GIECHE J. 2007. How to Assess Equine Oral Health. $53^{\text {rd }}$ Annual Convention Am. Assoc. Equine Practitioners. Orlando, Florida. Disponible desde Internet en: http://www.ivis.org/proceedings/aaep/2007/ gieche/chapter.asp (con acceso 12/07/08).

16. HABEL, R. 1988. Anatomía Aplicada Veterinaria. Ed. Acribia (España): p.8-13.

17. JONES, S. 2005. Enfermedades orales. En: Reed, S.; Bayly, W.; Sellon, D. eds. Medicina Interna Equina. Vol. 2. $2^{\mathrm{a}}$ ed. Ed. Intermédica (Argentina): p.937-939.

18. KILIC, S.; DIXON, P.; KEMPSON, S. 1997a. A light microscopic and ultrastructural examination of calcified dental tissues of horses. II ultrastructural enamel finding. Equine Vet. J. 29:198 -205.

19. KILIC, S.; DIXON, P.; KEMPSON, S. 1997b. A light microscopic and ultrastructural examination of calcified dental tissues of horses. III Dentine. Equine Vet. J. 29:206-212.

20. KIRKLAND, K.; BAKER, G.; MANFRA, S.; EURELL, J.; LOSONSKY, J. 1996. Effects of aging on the endodontic system, reserve crown, and roots of 
equine mandibular cheek teeth. Am. J. Vet. Res. 57:31-38.

21. KLUGH, D. 2006. Endodontic Considerations of Equine Incisor and Canine Teeth. Am. Assoc. Equine Practitioners. Disponible desde Internet en: http://www.ivis.org/proceedings/aaepfocus/2006/ klugh1.pdf (con acceso 12/07/08).

22. KONIG, H.; LIEBICH, H. 2005. Aparato Digestivo. En: Konig, H.; Liebich, H. eds. Anatomía de los Animales Domésticos. $2^{\mathrm{a}}$ ed. Ed. Panamericana (España): p.25-30.

23. LINKOUS, M. 2006. Dental Conditions Affecting the Juvenile Performance Horse (2-5 Years). Am. Assoc. Equine Practitioners. Disponible desde Internet en: http://www.ivis.org/proceedings/aaepfocus/2006/ linkous1.pdf (con acceso 12/07/08).

24. LOWDER, Q.; MUELLER, E. 1998. Dental embryology, anatomy, development and aging. Vet. Clin. North Am Equine Pract. 14(2):227-245.

25. MARTIN, M. 1999. A Systematic Approach to Estimating the Age of a Horse. Am. Assoc.Equine Practitioners Annual Convention. 45:273-275. Disponible desde Internet en: http://www.ivis.org/ proceedings/AAEP/1999/273.pdf (con acceso 12/07/08).

26. MARVIN, G. 1992. Odontología Equina. En: Robinson, E ed. Terapéutica Actual en Medicina Equina 2. Ed. Intermédica (Argentina): p.7.

27. MITCHELL, S.; MITCHELL, S.; KEMPSON, S.; DIXON, P. 2003. Structure of peripheral cementum of normal. Equine cheek teeth. J. Vet. Dent. 20:199-208.

28. MORALES, F. 1997. El Caballo. Ed. Marmor (Colombia): p.356-358.

29. MUYLLE, S. 2002. Determinación de la Edad. En: Baker, G; Easley, J. eds. Odontología Equina XXI. Ed. Intermédica (Argentina). p.39-51.

30. MUYLLE, S.; SIMOENS, P.; LAUWERS, H. 2002. A study of the ultrastructure and staining characte- ristics of the 'dental star' of equine incisors. Equine Vet. J. 34(3):230 -234.

31. PEÑA, F.; HERRADOR, P. 1991. Arcadas incisivas y edad en caballos de pura raza española. Cronología dentaria. Arch. Zootec. 40:181-192.

32. PIMENTEL, L. 2007. Intraoral extraction techniques in standing horse. Pesq. Vet. Bras. 27(Supl.):5758.

33. REAL, C. 1990. Zootecnia Equina. Ed. Trillas (Mexico). p.35-47.

34. RICHARDSON, J. 1997. Ageing horses an illustrated guide. In Practice. 19(9):486-489.

35. RICHARDSON, J.; CRIPPS, P.; LANE, J. 1995. An evaluation of the accuracy of ageing horses by their dentition: changes of dental morphology with age. Vet Rec. 137:117-121.

36. RICHARDSON, J.; LANE, J.; WALDRON, K. 1994. Is dentition an accurate indication of age of a horse? The Vet. Record. 135(2):31-34.

37. RUCKER, B. 1996. Incisor Procedures for Field Use. Am. Assoc. Equine Practitioners Proc. 42:22-25.

38. RUCKER, B. 2003. Enfermedades de la cavidad oral y el paladar blando. En: Mair, T.; Divers, T.; Ducharme, N. eds. Manual de Gastroenterología Equina. Ed. Intermédica (Argentina): p.81-83.

39. RUCKER, B. 2004. Incisor and molar occlusion: normal ranges and indications for incisor reduction. 50th Annual Convention of the American Association of Equine Practitioners, Denver, Colorado. Disponible desde Internet en: http://www. ivis.org/proceedings/AAEP/2004/Rucker1/chapter. asp?LA=1 (con acceso 14/07/08).

40. RUCKER, B. 2006. Dental Conditions Affecting the Geriatric Horse. Am. Assoc. Equine Practitioners. Disponible desde Internet en: http:/www.ivis.org/ proceedings/aaepfocus/2006/rucker1.pdf (con acceso 14/07/08). 
41. SANDOVAL, J. 1976. Anatomía del Caballo. Cabeza y Órganos de los Sentidos. Tomo III. Ed. Acribia (España).p.86-90.

42. SCOGGINS, D. 2001. Bits and bitting and dentristy. Proc. Am. Assoc. Equine Practitioners. 47:138141.

43. SCRUTCHFIELD, W. 1991. Incisors and Canines. Am. Assoc. Equine Practitioners. Disponible desde Internet en: www.aaep.org/eve/aug_01/alexander0801.pdf (con acceso 16/07/08).

44. SCRUTCHFIELD, W. 2006. Wolf Teeth: How to Safely and Effectively Extract and Is It Necessary. Am. Assoc. Equine Practitioners. Disponible desde Internet en: ttp://www.ivis.org/proceedings/ aaepfocus/2006/scrutchfield1.pdf (con acceso 17/07/08).

45. SCRUTCHFIELD, W.; SCHUMACHER, J.; MARTIN, M. 1996. Correction of Abnormalities of the Cheek Teeth. 42nd Annual Convention Am. Assoc. Equine Practitioners. 42:11-21.

46. TAYLOR, F.; HILLYER, M. 1999. Técnicas Diagnósticas en Medicina Equina. Ed. Acribia (España): p.21.
47. TOIT, N. 2006a. Age Related Changes in Dentition. Am. Assoc. Equine Practitioners. (USA). Disponible en URL: http://www.ivis.org/proceedings/aaepfocus/2006/dutoit1.pdf (con acceso 16/07/08).

48. TOIT, N. 2006b. Gross Equine Dentition and Their Supporting Structures. Am. Assoc. Equine Practitioners. Disponible desde Internet en: http://www. ivis.org/proceedings/aaepfocus/2006/dutoit2. pdf (con acceso 18/07/08).

49. TREMAINE, H. 1997. Dental care in horses. In Practice. 19(4):186-199.

50. VILLANUEVA-SALCEDO, E. 2001. Atención orodental en el equino de alto rendimiento deportivo. Rev. Sanit. Milit. Mex. 55(3):128-131.

51. WALMSLEY, J. 1993. Some observations on the value of ageing 5-7 year old horses by examination of their incisor teeth. Equine Vet. Educ. 5:295-298.

Recibido: Septiembre 20 de 2008

Aceptado: Marzo 26 de 2010 